\section{$\underset{\substack{\text { hommes } \\ \text { \& migrations }}}{ }$}

\section{Hommes \& migrations}

Revue française de référence sur les dynamiques

migratoires

\section{$1321 \mid 2018$}

Les mots de l'exil dans l'Europe du XIXe siècle

\title{
Les réfugiés en France
}

Au prisme des circulaires du ministère de l'Intérieur (1830-1870) : Pour une étude conjointe des discours et pratiques de l'administration

\section{Delphine Diaz}

\section{(2) OpenEdition}

\section{Journals}

Édition électronique

URL : http://journals.openedition.org/hommesmigrations/4204

DOI : 10.4000/hommesmigrations.4204

ISSN : 2262-3353

Éditeur

Musée national de l'histoire de l'immigration

Édition imprimée

Date de publication : 1 avril 2018

Pagination : $33-40$

ISBN : 978-2-919040-41-4

ISSN : $1142-852 X$

Référence électronique

Delphine Diaz, «Les réfugiés en France », Hommes \& migrations [En ligne], 1321 | 2018, mis en ligne le 01 avril 2020, consulté le 06 janvier 2021. URL : http://journals.openedition.org/hommesmigrations/ 4204 ; DOI : https://doi.org/10.4000/hommesmigrations.4204 


\title{
LES RÉFUGIÉS EN FRANCE
}

AU PRISME DES CIRCULAIRES DU MINISTÈRE DE L'INTÉRIEUR (1830-1870) : POUR UNE ÉTUDE CONJOINTE DES DISCOURS ET PRATIQUES DE L'ADMINISTRATION

Par DELPHINE DIAZ, maîtresse de conférences en histoire contemporaine, programme ANR Asileurope XIX, université de Reims Champagne-Ardenne.

\author{
Entre 1830 et 1870, I'arrivée en France d'étrangers venus de toute \\ l'Europe et de différents horizons idéologiques a conduit les \\ autorités françaises à encadrer leur accueil. La littérature \\ administrative de l'époque renseigne sur la construction de la \\ catégorie de réfugié. L'analyse des mots et des pratiques qui \\ leur sont associées dans les circulaires relatives aux réfugiés, \\ entre la monarchie de Juillet et la fin du Second Empire, permet \\ de mettre au jour ce langage du contrôle mobilisé par l'État pour \\ trier et classer les personnes qui bénéficient ou non de son \\ hospitalité.
}

En septembre 1834, Adolphe Thiers, alors ministre de l'Intérieur, diffusait auprès des préfets de départements une circulaire dans laquelle il mettait en avant la " générosité française », consistant à octroyer des secours à de nombreux réfugiés accueillis au milieu de circonstances «impérieuses ", mais à ses yeux «nécessairement temporaires ${ }^{1}$ ». La reconnaissance du titre de réfugié, qui s'accompagnait alors de l'attribution de subsides à l'étranger ainsi qualifié, était présentée par Thiers comme une " concession, et non (...) un droit acquis ${ }^{2} »$. Derrière le discours sur l'hospitalité française à l'égard d'étrangers persécutés, se déployait une volonté de contingenter l'accueil, selon une logique comptable et sans exclure une forme de méfiance vis-à-vis des motifs de ces émigrations.

Cet article se propose de mener une enquête sur les mots et les pratiques administratives adoptés face à l'accueil des « réfugiés », une catégorie d'étrangers en cours de définition dans la France du XIX siècle, mais aussi dans les autres pays d'asile européens, en particulier en Grande-Bretagne, en Suisse ou en Belgique $^{3}$. Une double focale est adoptée pour guider notre analyse : il s'agit, d'une part, d'interroger les dénominations, anciennes ou nouvelles, qui ont été employées pour désigner les étrangers venus s'établir en France sous la contrainte entre le début de la monarchie de Juillet et la fin du Second Empire. Comment les autorités administratives ont-elles de plus en plus pris le soin de distinguer plusieurs catégories d'étrangers, allant du voyageur au déserteur, en passant par l'ouvrier et le vagabond, et ont tenté de mieux isoler au sein de ces figures celle de létranger réfugié? Notre étude se propose d'autre part d'associer ces interrogations sur les mots et les catégories à une réflexion sur les 
pratiques administratives de l'État face à l'accueil des étrangers. Elle s'inscrit dans le sillage de travaux d'historiens, de sociologues et de politistes sur les marges de manœuvre dont disposent les autorités administratives dans la mise en œuvre des politiques migratoires. Les recherches de Gérard Noiriel sur les pratiques administratives face à l'immigration étrangère ont été pionnières de ce point de vue. Dans La tyrannie du national, celui-ci se proposait de faire une histoire sociale des pratiques administratives, en analysant les circulaires et instructions qui avaient progressivement renforcé le contrôle sur les étrangers du XIX ${ }^{\mathrm{e}}$ au XX $\mathrm{XX}^{\mathrm{e}}$ siècle ${ }^{4}$. Gérard Noiriel a également montré, à travers l'histoire des pratiques d'identification, combien les autorités administratives, à partir de la fin du XVIII siècle, ont de moins en moins dépendu des formes

L'appareil administratif était

certes moins développé

dans la France du XIXe siècle qu'aujourd'hui, mais il n'en reste pas moins que l'accueil des réfugiés a suscité, dès cette époque, l'apparition

de nouvelles structures destinées à leur réception. traditionnelles de reconnaissance des personnes, remplacées par de nouvelles méthodes, perfectionnées par les États modernes et leur bureaucratie en développement ${ }^{5}$.

Une telle perspective, tendant à interroger les pratiques administratives adoptées face aux étrangers ou aux naturalisés, a récemment inspiré le livre consacré par Claire Zalc aux dénaturalisations sous le régime de Vichy. Elle a mis en évidence la bureaucratisation des procédures, mais aussi la grande variété des attitudes suivies par les membres de la Commission de révision des naturalisations, depuis les preuves d'antisémitisme virulent jusqu'aux actes de résistance de ceux qui ont « multipli [é] les avis de maintien dans la nationalité française ${ }^{6}{ }$. Le temps présent inspire lui aussi des recherches nourries sur la liberté d'interprétation des textes dont font preuve les agents de l'administration préfectorale face à l'accueil des étrangers. Dans ses travaux sur la "politique du guichet », Alexis Spire a montré que, dans la France du début des années 2000, "l'extension du pouvoir discrétionnaire [a] affect[é] toutes les administrations chargées du contrôle de l'immigration ${ }^{7}$ » : leurs agents disposaient d'une marge de manœuvre importante, dépendant "étroitement des instructions de chaque chef de bureau ${ }^{8} "$.

\section{L'émergence d'une nouvelle catégorie administrative : le réfugié}

L’appareil administratif était certes moins développé dans la France du XIXe siècle qu'aujourd'hui, mais il n'en reste pas moins que l'accueil des réfugiés a suscité, dès cette époque, l'apparition de nouvelles structures destinées à leur réception. Sous la monarchie de Juillet, le ministère de l'Intérieur est devenu l'acteur central dans la politique de surveillance des réfugiés et d'attribution des secours, alors même que le ministère de la Guerre occupait une place prépondérante dans ce processus sous le Premier Empire et la Restauration. Néanmoins, même après 1830, le ministère de la Guerre a continué de se charger de la discipline des réfugiés militaires $^{9}$, ce qui supposait une coopération interministérielle et ne manquait pas de susciter des frictions.

L'organisation interne du ministère de l'Intérieur au début de la monarchie de Juillet apprend beaucoup sur l'intérêt accordé par le nouvel exécutif à la question des réfugiés, alors même que le nombre de ceux officiellement recensés et secourus par l'exécutif dépassait les 7200 personnes en $1832^{10}$. Entre le 17 mars 1831 et le 22 février 1836, le premier bureau de l'administration du ministère de l'Intérieur traitait systématiquement des réfugiés étrangers : ce premier bureau s'intitulait «Sûreté de l'État, réfugiés » jusqu'au 11 octobre 1832, puis

\footnotetext{
4. Gérard Noiriel, La tyrannie du national : le droit d'asile en Europe, 1793-1993, Paris, Calmann-Lévy, 1991. 5. Gérard Noiriel, "Vos papiers! ", in Genèses, $n^{\circ}$ 54, 2004, pp. 2-3. 6. Claire Zalc, Dénaturalisés. Les retraits de nationalité sous Vichy, Paris, Seuil, 2016, p. 318.

7. Alexis Spire, Accueillir ou reconduire. Enquête sur les guichets de l'immigration, Paris, Raisons d'Agir, 2008, p. 10. 8. Ibid., p. 11. 9. AD de Haute-Garonne, 4 M Étrangers 1, Circulaire du ministère de l'Intérieur du 13 août 1833 : "la discipline de ces dépôts [de réfugiés] continue à être du ressort de l'autorité militaire, la police politique (...) regarde [les préfets] ». 10. Archives parlementaires, t. 81, p. 613 : est évoqué un chiffre total de 7281 réfugiés subventionnés en 1832.
} 
jusqu’au 4 avril 1834 était baptisé « Police générale, réfugiés ", et enfin "Ordre public, réfugiés ", jusqu'au 22 février 1836. Ce n'est qu'après cette date que la question des réfugiés n'a plus été traitée par le premier mais seulement par le troisième bureau du ministère, la "police administrative » étant désormais plus clairement distinguée de la surveillance des réfugiés jusqu'à la fin de l'année $1839^{11}$.

Ces changements de dénomination et d'organisation ministérielle témoignent ainsi d'une urgence moindre accordée à la question de l'accueil et de la surveillance des réfugiés en France, après une première décennie du régime durant laquelle le pays a été parcouru par les réfugiés espagnols, italiens et polonais. Plus tard, au début du Second Empire, alors que le ministère de l'Intérieur est un temps remplacé par un ministère de la Police générale, le premier bureau sera de nouveau en charge de la " police des étrangers réfugiés ", signe que leur surveillance redevient une priorité politique ${ }^{12}$.

Les différents bureaux en charge des réfugiés entre 1830 et 1870 ont diffusé avec constance auprès des préfets de départements des circulaires concernant cette catégorie particulière d'étrangers, épaisse correspondance disséminée entre divers fonds d'archives, au sein de laquelle 241 textes ont pu être transcrits et rassemblés ${ }^{13}$. En retenant de telles bornes chronologiques pour délimiter ce corpus, il s'agissait d'inclure dans le périmètre de notre étude trois régimes différents - monarchie de Juillet, Seconde République, Second Empire - afin de mesurer les variations que l'on peut percevoir selon le contexte institutionnel et politique. Pour rassembler ces textes, il a fallu confronter les recueils de circulaires du ministère de l'Intérieur qui ont fait l'objet de publications, au demeurant très lacunaires, avec la lecture des sources conservées dans les fonds des Archives départementales ${ }^{14}$, mais aussi des Archives nationales de France et des

Archives nationales de l'Outre-mer pour ce qui concerne l'Algérie, afin de restituer une littérature grise administrative tombée dans l'oubli.

Recueillir ces textes réglementaires sur les réfugiés permet de cerner l'émergence d'une catégorie administrative en construction, à une époque qui s'est ouverte avec une première loi sur les «étrangers réfugiés » : adopté le 21 avril 1832, ce texte ne prenait pas soin de définir juridiquement les contours du groupe ainsi dénommé. Les trois régimes étudiés ont été inégalement pourvoyeurs de circulaires relatives aux réfugiés : sur les 241 textes analysés, 142 ont été édictés sous la monarchie de Juillet (soit près de 8 par an en moyenne), 69 sous la Seconde République (13,8 en moyenne par an) et 30 sous le Second Empire (seulement 1,6 en moyenne par an). La cadence avec laquelle ont été diffusées les circulaires traduit l'urgence qu'a représentée, à certains moments, l'accueil des réfugiés, mais elle reflète aussi l'intérêt inégal accordé par le pouvoir à la question de l'asile politique. Ainsi la Seconde République (février 1848-décembre 1852) se distingue-t-elle comme un temps fort: en près de cinq ans de régime, ont été adoptées 69

L'organisation interne du ministère de l'Intérieur au début de la monarchie de Juillet apprend beaucoup sur l'intérêt accordé par le nouvel exécutif à la question des réfugiés, alors même que le nombre de ceux officiellement recensés et secourus par l'exécutif dépassait les 7200 personnes en 1832 des 241 circulaires analysées sur une période de quarante années. En revanche, durant le Second Empire, le rythme de publication des circulaires sur les réfugiés s'est fait plus sporadique et, dans le même temps, les textes envoyés aux préfets se sont avérés de plus en plus répétitifs : ils se bornaient à rappeler la nécessité d'une « révision du contrôle des réfugiés subventionnés ${ }^{15}$ ", dans l'optique d'une réduction de leur nombre.

L'emploi même des termes utilisés pour désigner les étrangers venus en France sous la contrainte 
appelle des remarques liminaires. Si des expressions telles qu' « étrangers réfugiés » ou "réfugiés politiques » sont abondamment employées, d'autres mots, en revanche, demeurent singulièrement absents, comme celui d' " exilé ", pourtant très utilisé dans la littérature de l'époque, mais qui n'appartenait guère au vocabulaire administratif. Seul le syntagme «le temps de l'exil » est utilisé à deux reprises - en août $1837^{16}$ puis en juillet $1843^{17}$, à des dates où les tarifs des secours ont été abaissés -, non pas tant pour déplorer le temps d'isolement et d'éloignement vécu par ces étrangers, que pour souligner la nécessaire mise à
Si des expressions telles ou « réfugiés politiques»

sont abondamment employées, d'autres mots, en revanche, demeurent singulièrement absents, comme celui d'u exilé », pourtant très utilisé dans la littérature de l'époque, mais qui n'appartenait guère au vocabulaire administratif. qu'« étrangers réfugiés » profit de ce séjour forcé en France par les réfugiés, qui sont fortement incités à trouver des revenus ou à suivre des études dans les facultés qui leur étaient alors ouvertes. À travers la terminologie employée par la littérature grise des circulaires, s'affirme la ferme volonté de distinguer le réfugié d'autres figures d'étrangers qui se dessinent en filigrane comme autant de doubles négatifs : le déserteur ${ }^{18}$, surtout, mais aussi le vagabond, l'ouvrier ou « l'émigrant » étrangers, qui ne chercheraient à venir en France que pour des raisons économiques et non politiques. Soulignons néanmoins que, dans les moments où les autorités veulent refermer les frontières, le ministère tend précisément à nier ce qualificatif de "réfugié » à des individus qui cherchent pourtant sous la contrainte à gagner le sol français. En mai 1849, une circulaire qualifie d' "émigrants " près de 200 exilés polonais qui ont quitté de force le port de Trieste, dans l'Empire d'Autriche, à bord d'un navire à destination des États-Unis, et refuse l'accès au territoire de la République à ces victimes d'une "proscription récente ${ }^{19}$ ". L'année suivante, une nouvelle circulaire du ministère de l'Intérieur exhorte encore les préfets à mieux distinguer les réfugiés des "déserteurs et des mendiants, des vagabonds et des condamnés libérés ${ }^{20} "$.

\section{Le classement politique des origines migratoires}

Les étrangers auxquels les circulaires octroient le titre alors recherché de "réfugiés » sont loin de former un groupe uniforme. Outre les lignes idéologiques qui les partagent et les opposent entre eux, les clivages nationaux sont présents à l'esprit des autorités administratives. On parle ainsi fréquemment des «émigrations " pour renvoyer aux divers groupes nationaux que forment les colonies de réfugiés. D'ailleurs, dès le début de la monarchie de Juillet, le ministère de l'Intérieur cherche à distinguer les réfugiés selon leur nationalité. En avril 1833, le comte d'Argout, alors ministre de l'Intérieur, envoie aux préfets un modèle de comptabilité des réfugiés recevant des subsides, qui les distingue d'emblée par nation : «Ce compte présentera la situation numérique, par nation, de chaque classe de réfugiés; la quotité des secours qui leur sont alloués d'après le nouveau tarif. Le résultat figurera dans la colonne intitulée: "total de l'effectif par nation ${ }^{21}$ " ". Certes, le terme "nationalité » ne fera son entrée dans le vocabulaire législatif qu'en 1889 , avec l'adoption du « code de la nationalité » sous la Troisième République ${ }^{22}$. Mais de la monarchie de Juillet au Second Empire, il s'agit d'ores et déjà d'une catégorie administrative à laquelle le 
ministère de l'Intérieur se réfère prioritairement pour le classement et le fichage des étrangers.

Dans le corpus de circulaires analysé, les réfugiés espagnols - qu'ils soient libéraux, carlistes ou républicains - apparaissent indéniablement comme le premier motif de préoccupation pour le «bureau des réfugiés » du ministère de l'Intérieur. 109 des 241 circulaires étudiées les évoquent, dont 95 s'intéressant spécifiquement à leur sort. Viennent ensuite les Polonais : malgré leur grand nombre et la pérennité de leur séjour en France après 18311832, et en dépit des nouvelles émigrations de 1846 et de 1863-1864, ils éveillent moins d'inquiétudes de la part du ministère. 33 circulaires au total abordent le cas des Polonais, perçus comme un groupe moins problématique que les Espagnols car moins fracturé et surtout mieux accepté par la société française, comme le met en valeur un texte de septembre 1834 : "Les Polonais, quoique plus récemment expatriés [que les Italiens], n'ont pas eu moins de chances d'établir d'utiles rapports. Favorisés par la sympathie nationale, ils ont été accueillis avec bienveillance. Ils se sont familiarisés avec notre langue ; plusieurs tirent des avantages de l'exercice de leurs professions $s^{23}$. "

L'émigration politique italienne, plus ancienne en France - sous la Restauration, nombre de libéraux originaires du Royaume des Deux-Siciles ou du Piémont-Sardaigne avaient rejoint le pays qui avait pourtant envisagé de fermer ses frontières aux carbonari -, est évoquée dans quinze circulaires seulement. Quant aux réfugiés allemands, qui font l'objet de neuf textes, il s'agit là encore d'un groupe particulièrement hétérogène, au sein duquel les Badois concentrent toutes les attentions à partir de mars 1848. Pourtant nombreux en France mais n'ayant pas fait l'objet de secours, les réfugiés d'origine germanique de la période du Vormärz ${ }^{24}$ ne sont jamais mentionnés par le ministère, et l'émigration allemande n'apparaît donc comme un objet de préoccupations quavec le printemps des peuples. L'année 1849 est marquée par la volonté de réexpédier chez eux les réfugiés autorisés par le GrandDuché de Bade à y retourner, mais ceux-ci sont accusés de préférer « rester en France où ils sont secourus, que de rester dans un pays où ils manqueraient de ressources ${ }^{25}$ ", preuve que la frontière entre le réfugié et l'émigrant est alors jugée perméable.

Si la figure du réfugié est loin d'être homogène, puisqu'elle s'avère à travers ces textes segmentée selon les opinions politiques et les origines nationales, on peut aussi s'interroger sur le genre de ce "réfugié étranger », toujours décliné au masculin. Alors que la présence des femmes parmi les émigrations politiques accueillies dans la France du XIX siècle est attestée ${ }^{26}$, comment se trouve-t-elle reflétée par les circulaires du ministère de l'Intérieur? Seuls cinq textes du corpus - quatre portant sur les
Si la figure du réfugié est loin d'être homogène, puisqu'elle s'avère à travers ces textes segmentée selon les opinions politiques et les origines nationales, on peut aussi s'interroger sur le genre de ce « réfugié étranger », toujours décliné au masculin. sous la monarchie de Juillet, l'autre sur les Polonaises au printemps $1848^{27}$ - se consacrent spécialement aux femmes, tandis que douze autres abordent incidemment leur situation. La figure du réfugié demeure ainsi presque exclusivement masculine et les femmes accompagnant les réfugiés secourus se trouvent invisibilisées : seule la référence à la fonction de " chef de famille » ou de « père de famille " assumée par les hommes laisse entendre que ceux-ci sont venus en France accompagnés ou ont été suivis dans leur migration, que ce soit par leurs femmes ou leurs enfants.

Bien que les circulaires demeurent le plus souvent silencieuses sur le statut des femmes réfugiées, la cellule familiale et le maintien de son intégrité dans la migration ne sont pas indifférents aux autorités françaises, qui adoptent pour principe, en 
mars 1841, de ne pas séparer «les individus composant une même famille, à moins d'une nécessité abso$l u e^{28} »$. Le fait d'avoir une charge de famille confère un statut relativement protecteur aux réfugiés qui, au début du Second Empire, sont nombreux à se voir radier des listes de secours par un régime peu favorable à l'asile. Une circulaire de novembre 1854 préconise de supprimer les secours à ceux qui «n'ont aucune charge de famille " et qui, "à raison de leur aptitude au travail », peuvent en priorité "cesser de participer à l'assistance de l'État ${ }^{29}$ ». À la fin des années 1850, à un moment où la vision comptable prévaut dans la gestion des étrangers secourus, le réfugié jeune et célibataire demeure dans le viseur du ministère de l'Intérieur, puisqu'il est considéré comme étant le plus apte à se procurer «des ressources par le travail ${ }^{30} »$.

\section{Le pouvoir discrétionnaire du langage à l'égard des réfugiés}

L'analyse des mots confrontée à celle des pratiques administratives adoptées à l'égard des réfugiés conduit à interroger le pouvoir discrétionnaire des gouvernants, qui, comme l'a rappelé Alexis Spire, peut être entendu de deux manières. D'une part, comme leur "capacité (...) à transcrire un texte juridique à destination des agents qui vont l'appliquer », en particulier à travers des circulaires ou des règlements qui permettent de rendre la loi intelligible et applicable. Dans sa seconde acception, le pouvoir discrétionnaire renvoie à une "décision particulière prise par un agent pour appliquer une norme juridique à un cas singulier : accorder une autorisation, octroyer certaines prestations ou encore concéder une faveur ${ }^{31}$ ". Les circulaires étudiées, censées traduire la position du ministère de l'Intérieur vis-à-vis des réfugiés, en permettant aux préfets d'appliquer la loi initialement conçue comme temporaire du 21 avril 1832, puis toutes celles qui suivront ${ }^{32}$, n'ont pas empêché pour autant certains préfets de s'écarter des prescriptions qui leur étaient faites. En mai 1833, le comte d'Argout, alors ministre de l'Intérieur, fait état des «inconvénients plus ou moins graves » résultant de l'action de préfets qui ont négligé d'envoyer à leurs collègues des instructions pour surveiller sur leur route des réfugiés «qui doivent quitter la France $^{33}$ ", autrement dit des expulsés. Peu de temps après, dès juillet 1833 , le ministre doit de nouveau adresser ses remontrances aux préfets qui ont manqué aux règles élémentaires relatives à l'assignation à résidence des réfugiés : certains d'entre eux ont été dirigés vers des villes où la première instruction relative aux réfugiés leur interdisait pourtant formellement de résider ${ }^{34}$.

Le point de vue des préfets, des sous-préfets, mais aussi des maires des villes concernées par l'accueil des réfugiés révèle leurs marges de manœuvre dans l'encadrement de ces populations étrangères, tout autant que le désarroi qu'ils éprouvaient en mettant en œuvre certaines décisions de l'exécutif. Deux éléments de la politique de contention adoptée à l'égard des réfugiés en France faisaient régulièrement l'objet de discussions : l'assignation à résidence et l'application d'arrêtés d'expulsion. Tandis que le principe de l'internement dans les dépôts, devenu de plus en plus souple avec le temps, pouvait faire l'objet de dérogations facilitées ou entravées par les préfets, l'application des arrêtés d'expulsion constituait un autre espace de liberté pour les préfets et les maires, parfois amenés à contrevenir aux ordres venus de Paris. Le département du Nord, frontalier de la Belgique, constitue à cet égard un observatoire des hésitations et des incompréhensions suscitées par l'application de cet 
outil du contrôle migratoire qu'était l'expulsion. En mai 1849, le sous-préfet de Valenciennes s'alarme des conséquences des arrêtés d'expulsion adoptés à l'encontre de réfugiés polonais dirigés vers la Belgique, qui pourtant se voient systématiquement refoulés une fois arrivés à la frontière. Il signale alors au préfet du Nord leur état d'épuisement et cherche à faciliter leur entrée à l'hôpital de Valenciennes pour une durée de 24 heures $^{35}$. En 1855, au début du Second Empire, alors que son successeur est confronté au même problème du retour de réfugiés expulsés depuis la France vers la Belgique, et pourtant refoulés à la frontière francobelge, à Quiévrain, le sous-préfet de Valenciennes, prend cette fois le parti de diriger ces indésirables directement vers l'Angleterre, via Calais ${ }^{36}$. Dans cette variété des réactions adoptées, se situe la latitude dont disposaient alors les représentants de l'administration préfectorale.

\section{Une circulation des mots et des pratiques administratives à l'échelle européenne}

Les mots et les pratiques administratives qui ont été forgés dans la France du XIX`e siècle pour dénommer et encadrer les réfugiés ont circulé en Europe. Sans évoquer le cas de la Grande-Bretagne, qui, entre 1826 et 1905, accueillait les étrangers sans la moindre sélection, deux autres pays francophones et frontaliers qui jouaient aussi un rôle de terre d'asile privilégiée - la Belgique après son indépendance et la Confédération helvétique - ont adopté et adapté certaines des mesures administratives appliquées aux réfugiés en France. Idesbald Goddeeris a montré que, peu après l'arrivée en Belgique de Polonais de la "Grande Émigration », au nombre d'environ deux cents à partir de l'hiver
1831-1832, le principe de l’assignation à résidence des réfugiés dans des " dépôts » avait été un temps envisagé par les autorités belges, en 1833, avant d'être abandonnés7. Deux ans plus tard, le pays adoptait un premier texte sur les étrangers, la loi du 22 septembre $1835^{38}$ qui disposait dans son article 5 que «le gouvernement pourra[it] enjoindre de sortir du territoire du royaume létranger qui quittera [it] la résidence qui lui aura[it] été assignée ${ }^{39}$ ». On ne peut que souligner combien cette formule se rapproche de celle de la loi française du 21 avril 1832 sur les étrangers réfugiés, elle aussi pensée comme temporaire, selon laquelle le gouvernement pouvait astreindre les réfugiés à se rendre dans la ville «qui leur sera[it] indiquée » et à les "enjoindre de sortir du royaume, s'ils ne se rend[ai]ent pas à cette destination".

En Suisse, lieu d'asile rejoint par les réfugiés espagnols, italiens ou polonais dans les années 1830, point de loi s'inspirant de la législation française sur les réfugiés, mais l'application de "mesures de sûreté ». Parmi celles-ci, l’expulsion était privilégiée, notamment pour éloigner les membres de la « Jeune Europe » qui avaient participé en 1834 à la tentative d'invasion de la Savoie. Le directeur de la police centrale de la République de Berne précise en juillet 1836 que les réfugiés politiques " agitateurs » doivent être «arrêtés », puis « expédi [és] au-delà de la frontière ${ }^{40} »$. Avec le printemps des peuples et l'arrivée en grand nombre sur le territoire de la Confédération de réfugiés badois, puis de proscrits républicains français, l'assignation à résidence de ces étrangers remuants apparaît comme une mesure incontournable pour garantir l'ordre public en Suisse. Des circulaires des années 1850-1851 attestent que des réfugiés étrangers sont "assignés " à résidence dans certains cantons helvétiques et que ce terme est employé de manière commune par les autorités de police, qui peuvent 
aussi parler de réfugiés "adjugés aux cantons ». Une ordonnance adoptée dans le canton de Berne le 28 août 1850 concernant la «tolérance des réfugiés politiques " évoque ainsi les

Si l'asile a été largement accordé en France à des étrangers venus de toute

l'Europe et de différents

horizons idéologiques,

l'analyse de la littérature grise administrative met en évidence les modalités par

lesquelles le ministère de I'Intérieur entendait classer, encadrer, protéger, mais aussi trier, radier et expulser les réfugiés secourus. "réfugié[s] adjugé[s] au canton de Berne par les autorités fédérales $^{41} »$ : pour pouvoir y demeurer, ceux-ci doivent recevoir de la Direction de la police centrale du canton un "acte de tolérance» portant leur nom, et «indiquant expressément la date de l'arrêté " qui les aura imposés au canton, l'autorité dont ils émanent, ainsi que la localité où le porteur de l'acte doit séjourner. Si cette pièce ne porte pas le nom de «bulletin individuel de réfugié ", comme c'est le cas en France à partir de mars $1833^{42}$, elle s'en rapproche du point de vue des informations qui doivent y figurer.

\section{Conclusion}

En explorant les circulaires relatives aux réfugiés de la monarchie de Juillet jusqu'à la fin du Second Empire, on a voulu mettre au jour les dénominations, mais aussi les outils d'identification et de contrôle élaborés pour circonscrire une catégorie d'étrangers en cours de définition. Si l'asile a été largement accordé en France à des étrangers venus de toute l'Europe et de différents horizons idéologiques, l'analyse de la littérature grise administrative met en évidence les modalités par lesquelles le ministère de l'Intérieur entendait classer, encadrer, protéger, mais aussi trier, radier et expulser les réfugiés secourus. Elle met en valeur les temporalités de l'asile, de l'urgence de l'accueil des réfugiés sous la Seconde République, jusqu'au triomphe, sous le Second Empire, d'une logique policière, cherchant à expulser les réfugiés démocrates, tout autant que d'une logique comptable, visant à radier de plus en plus d'étrangers des listes de secours.

Dans les circulaires diffusées tout au long des trois régimes envisagés, le ministère de l'Intérieur accorde tout son intérêt aux circulations européennes des réfugiés établis en France de manière plus ou moins pérenne. En septembre 1851, quelques mois avant le coup d'État de LouisNapoléon Bonaparte, le ministre Léon Faucher note que beaucoup de réfugiés "se rendent soit en Angleterre, soit dans d'autres pays de l'Europe, et reviennent ensuite chez nous reprendre le cours de leurs manœuvres », déplorant ces "déplacements continus » qui se rattachent selon lui aux «projets révolutionnaires préparés à Londres, à Genève, en Italie et surtout en France ${ }^{43}$ ». Ces mobilités transnationales de réfugiés dans l'Europe du XIXe siècle méritent indéniablement d'être retracées et cartographiées. Mais, accompagnant les déplacements continus de réfugiés, ce sont aussi les dénominations et les pratiques administratives forgées pour les identifier et contraindre leurs mouvements qui ont franchi les frontières des pays européens. 